\title{
High Compression Ratio Image Processing Techniques using combinations of WT and IFS
}

\author{
Y.G. Zhang \\ Institute of Systems \\ Science, Academia \\ Sinica, Beijing, China
}

\author{
X.J. Li \\ Dept. of Computer Scie. \\ The Beijing University of \\ Aeronautics \& Aerospace.
}

\author{
T.S. $\mathrm{Ng}$ \\ Dept. of E. \& E. Engin. \\ The Uni. of Hong Kong, \\ Hong Kong
}

\begin{abstract}
Many works of compressing image based on Wavelet transformation have been presented in recent years. Also, the method from Fractals is used in this field. First one is using of the pyramid subband decomposition, second takes advantage of self-similarity between the basic image and subimages. We combine these two kind of methods as a hybrid algorithm to complete compression of image. The experiments shown that this method has better performance than many others, especially, in the high compression ratio situation. A comparison with the famous EZW algorithm shows that its performance is close to the EZW algorithm.
\end{abstract}

\section{Introduction}

The technique of image compression has been quickly developing since JPEG was proposed. Especially, in recent years many works of image compression based on Wavelet Transformation and Fractals respectively reported. Definitely, The wavelet and Fractals are very useful tools for image compression. In the predictable future the JPEG must be replaced by other standard. The reason is that if we want to get high compression ratio with acceptable image quality, the JPEG in which the main technique of encoding is Discrete Cosine Transformation (DCT) is impossible according to amount of experiments. Many works based on wavelet transformation shown the result that in the case of compression ratio is less than 16 then there are not obvious difference between the reconstructed images using of DCT and WT respectively, but in the case of compression ratio is bigger than 30 the differences between reconstructed images are quite visionable. JPEG has two main drawback, one is block effects which is from DCT, the another is big errors due to Vector Quatization (VQ). DCT is operated on the small block of $8 \times 8$ pixels of the image, this is a spatial transformation, but not in the frequency area. However, the wavelet transformation is a spatial--frequency transformation. It operates on the whole image and results in the components in different bandwidth. VQ essentially is a finite classification of m-dimensional sample space. In order to give a finite good classification with small diameter in each class, that means small distortion in the reconstructed image, then the codebook will be very huge. In fact, there is a big redundancy of codewords for a specific image. Basically VQ is a probabilistic type encoding, so it is a better encoding method if we know the probabilistic distribution of the images. As mentioned in many works, that is impossible to know the probabilistic distribution of the images. So, we need other encoding method for image compression. Fractals is a very useful tool to deal with the self-similarity problem. If we ignore the time-consuming searching process, the encoding method is more reasonable than VQ. So far, the EZW (Zero Tree Wavelet) [1] method is the best in the high compression ratio situation that the reduction of PSNR is almost linear with increase of compression ratio. We have repeated the experiment and have got very close result (see section 5). In this paper we propose a hybrid algorithm to complete compressing image which combine the both wavelet transform and the fractals. The result show us that it has still better performance in high compression ratio situation. 


\section{Subband decomposition based on Wavelet Transformation [2]}

The pyramid decomposition of image by using of wavelet transformation is well-known. The subimages in the subbands are similar to the original image in the sense that the subimage in the most coarser subband is similar to the original and reduction of size is proportional, the subimage in the other subbands which represent the edges information of image are still similar to original and those in other subbands. So far, the subbands encoding methods use only the subband frequencies information included in the decomposition based on the wavelet transformation, but not the both subbands decomposition and similarity between the subimages.

\section{The compression method based on}

\section{IFS [3]}

Fractals is also a tool that can be used as to compress an image based on the IFS (Iterative Function System). Basically, it includes a group of affine transformations which describe the similarities between the basic picture components and the subimages with any size in the original images. The affine transformation can describe many relations of similarities such as dilation, rotation, affine. If a subimage similar to a basic picture component, then the corresponding coefficients in the affine transformation is the encoding for that subimage. Usually, the design of IFS is the basic problem for compression by using of fractals. Due to a huge amount of searching calculation, so the IFS algorithm always a timeconsuming job. To reduce the time consumed a prior information are needed. For example, if we know the subimage has no any rotation relative to the original image or subimages are proportional to the original image in their size without affine relation, then the amount of searching will be reduced. This is just the situation for wavelet transformation, the subimages from wavelet transformation have no any rotations and affine relations relative to the original image, the size of subimages is proportional to the original image and have the same direction. So, when the IFS operates on the pyramid of image subband composition by using of wavelet transformation the amount of searching is quite reduced.

\section{The hybrid compression technique}

Based on what mentioned above we have a very clear thinking that the pyramid resulted in from wavelet transformation has two features: one is the subbands decomposition of original image; another is the self-similarity between the subimages and the original image. Our research result is to propose some new algorithms that combined the two features and give high compression ratio of image with better quality.

\section{Algorithm: (Hybrid compression)}

step 1. Take wavelet transformation onto the original image for adequate times, for example, 5 times which is supposed as $512 \times 512 \times 8$ monochrome. The picture transformed is called as $T$-picture with 5 levels. The coarsest subimage is called as the level 1.

Step 2. Chose the coarsest subimage in the level 1 of the T-picture as a standard and split the three subimages in the level 2 into $4 \times 4$ small blocks, and compress them by using IFS, if error in some block is $>$ threshold, this means it has no similarity with the standard and can not be compressed, then save it;

Step 3. Split the three subimages in the level 3 into $8 \times 8$ small blocks and compress them by using IFS, if error in some block is $>$ threshold, then compress the block by using JPEG; if the error is still > threshold, then split this $8 \times 8$ small block into $4 \times 4$ even more small blocks and compress them by using IFS; if error is still > threshold still, then save it;

step 4. Split the three subimages in the level 4 into $16 \times 16$ small blocks and compress them by using IFS, if error in some block is > threshold, then split 
this block into $8 \times 8$ small blocks and compress them by using IFS; if the error in some $8 \times 8$ block is still $>$ threshold, then compress the block by using JPEG. If the error of it is still $>$ threshold, then split this $8 \times 8$ block into $4 \times 4$ more small blocks and compress them by using IFS, if error in some $4 \times 4$ block is still $>$ threshold, then save it;

step 5. Split the three subimages in the level 5 into $32 \times 32$ small blocks and compress them by using IFS, if error in some block is $>$ threshold, then split it into 16x16 small blocks and compress them by using IFS. If error in some block is > threshold, then split it into $8 \times 8$ blocks. If the error in some $8 \times 8$ block is $>$ threshold, the compress it by using JPEG. If the error is still $>$ threshold, then split it into $4 \times 4$ small blocks and compress them by using IFS, if error in some $4 \times 4$ block is $>$ threshold, then save it.

\section{Experiment results and comparison [5]}

We have done several experiments to check the method proposed by us. We have compressed different images which are used often by other Authors. Here we show the result on the picture named LENA, the real image is a monochrome picture of $512 \times 512 \times 8$. One of experiment is for repeating the EZW algorithm, the result is shown on the table 1 and named A-EZW. The original result of EZW by Shapiro is listed also in the table. One can find that they are very close. The algorithm in this work is named Hybrid, the real pictures compressed by it is shown in the Fig. 1 in the last page, and result is listed in Table 1. It is little worse than EZW and A-EZW. The comparison of Hybrid, A-EZW, EZW and JPEG is illustrated in Fig. 2. It is easy to see that in high compression ratio situation EZ,W and Hybrid is much better than JPEG (in fact also many others).

TABLE 1 Experiment results (PSNR--dB)

\begin{tabular}{r|rrcr} 
CR & Hybrid & JPEG & EZW & A-EZW \\
\hline 8 & 37.6 & 38.1 & 39. & 39.4 \\
16 & 34.9 & 34.8 & 36.3 & 36 \\
32 & 31 & 30.5 & 33.2 & 32.1 \\
64 & 27.2 & 22 & 30.3 & 29.2 \\
128 & 24.8 & 10.5 & 27.8 & 26
\end{tabular}

Comparison of Hybrid, A-EZW, EZWand JPEG

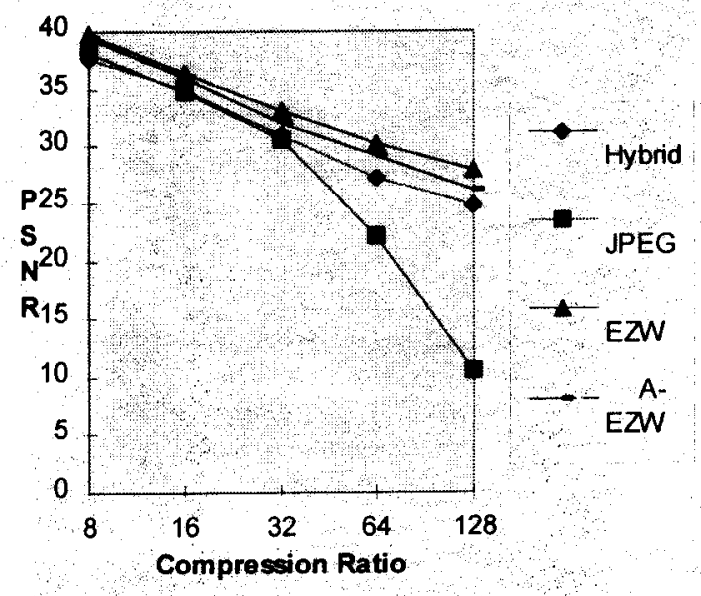

Fig. 2 The illustration of comparison

\section{References}

[1] Shapiro,J.M, "Embedded Image Coding Using Zerotree of Wavelet Coefficients", IEEE transactions on Signal Processing, Vol.41, No.12, Dec. 1993, pp.3445-3462.

[2] Woods, J W,ED., "Subband image coding", Boston, MA: Kluwer, 1991.

[3] Grochenig,K,, and Madych, W.R., "Multiresolution Analysis, Haar Bases, and Self-similarTilings of $\mathrm{R}^{\mathrm{n} \text {, }}$, IEEE Transaction on Information Theory, Vol.38, No.2, March 1992, pp.556--568.

[4] Wornell, G.W., and Oppenheim, A.V., "WaveletBased Representations for a class of self-similar signals with Application to Fractals Modulation", IEEE Transaction on IT, Vol.38, No2, March 1992, pp. 785- -800 .

[5] Hilton, H, "Still ang Moving Pictures compression", Multimedia System, No.1., 1995

[6] "Fractals and Wavelet", (1993)(call 530.15615 L9)

[7] "Fractals in Engineering", (1994)(call 620.001514 F7)

[8] "Fractals for the classroom", (1994)(call 514.74 P37)

[9] "Iterative Function System", (1994)(call 621.367 P47) 


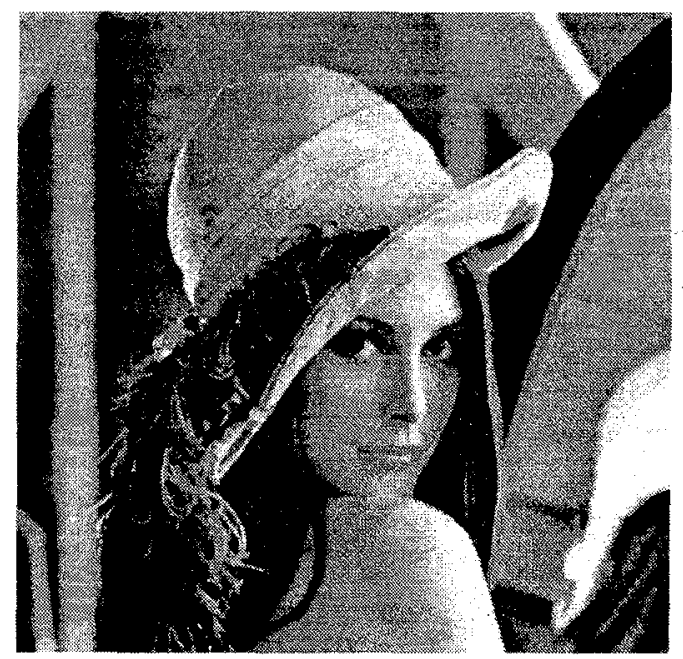

(a) The Original LENA

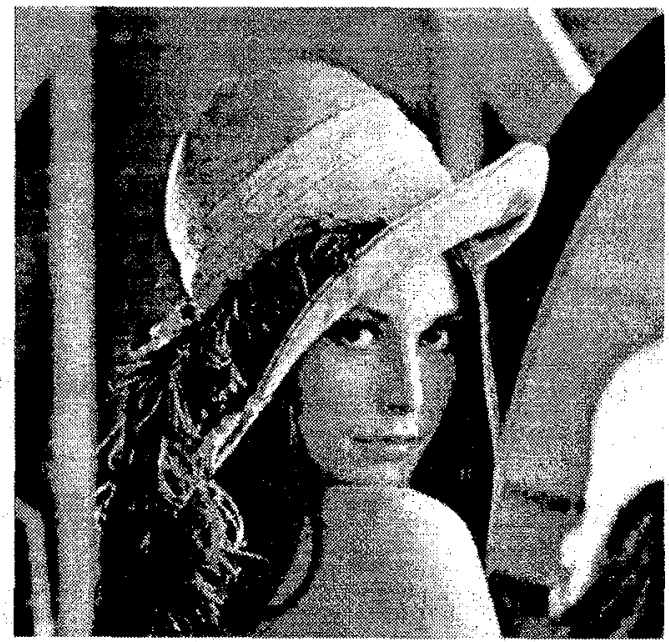

(c) $\mathrm{CR}=16$

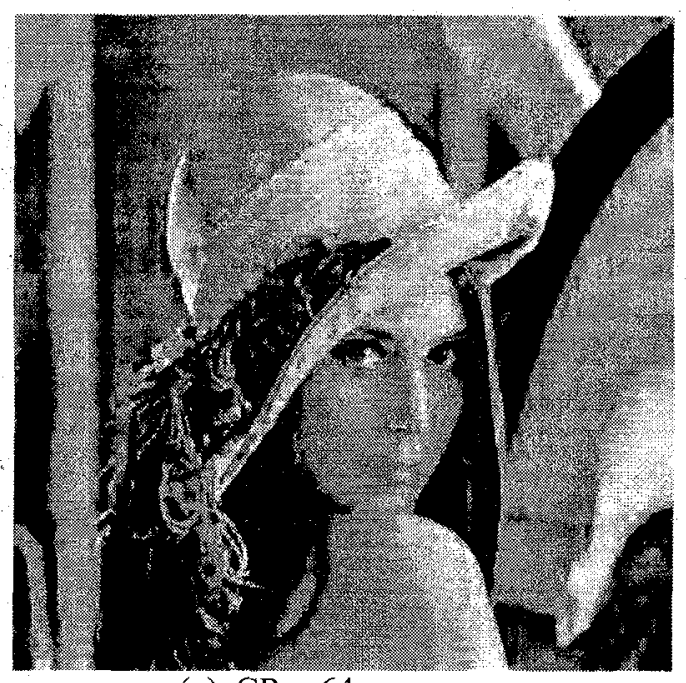

(e) $\mathrm{CR}=64$

Fig.1 The compression results by using of Hybrid algorithm. The original Lena is $512 \times 512 \times 8$.

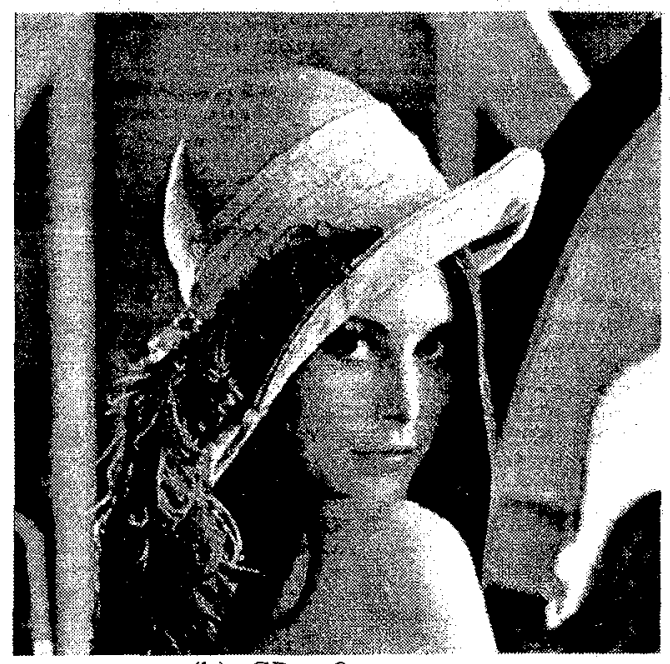

(b) $\mathrm{CR}=8$

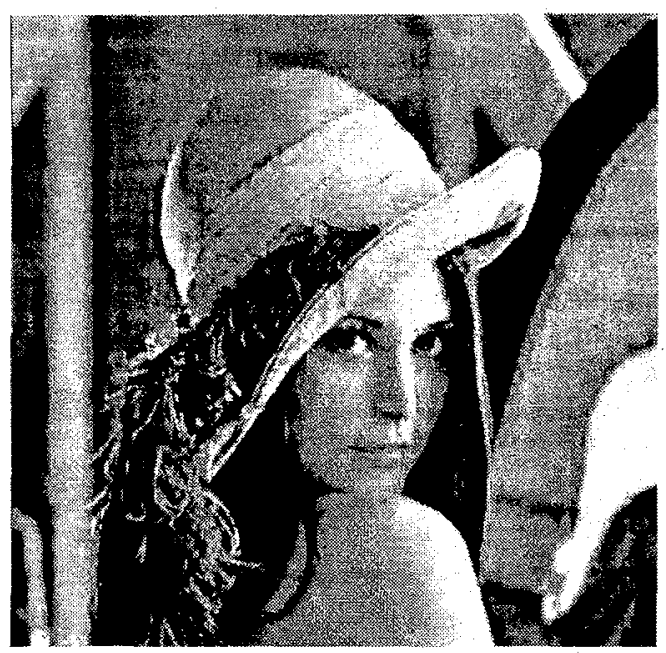

(d) $\mathrm{CR}=32$

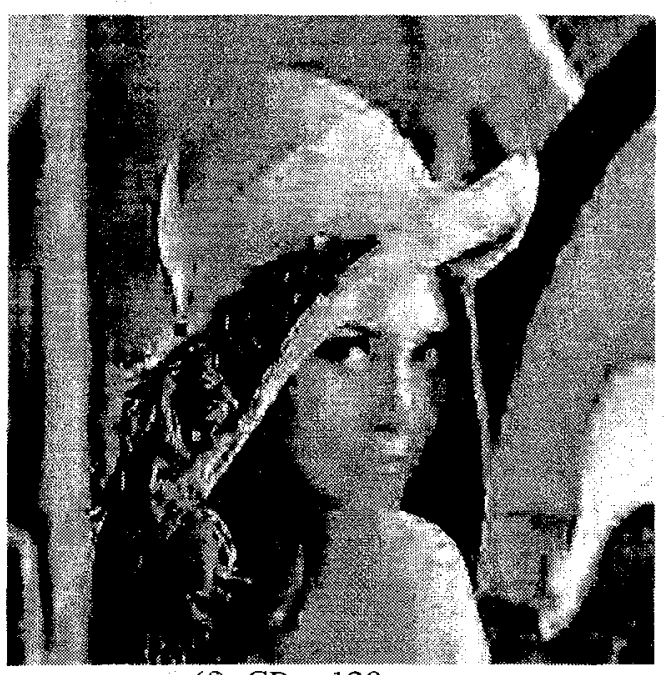

(f) $\mathrm{CR}=128$ 